PROCEEDINGS OF THE

AMERICAN MATHEMATICAL SOCIETY

Volume 133, Number 9, Pages 2783-2793

S 0002-9939(05)07823-8

Article electronically published on April 19, 2005

\title{
ON EMBEDDINGS IN THE SPHERE
}

\author{
JOHN R. KLEIN
}

(Communicated by Paul Goerss)

\begin{abstract}
We consider embeddings of a finite complex in a sphere. We give
\end{abstract} a homotopy-theoretic classification of such embeddings in a wide range.

\section{INTRODUCTION}

Let $K$ be a connected finite complex. An embedding up to homotopy of $K$ in $S^{n}$ consists of a pair

$$
(M, h)
$$

where

- $M^{n}$ is a compact codimension zero PL submanifold of $S^{n}$;

- $\pi_{1}(\partial M) \rightarrow \pi_{1}(M)$ is an isomorphism;

- $h: K \rightarrow M$ is a simple homotopy equivalence.

Two embeddings up to homotopy $\left(M_{0}, h_{0}\right)$ and $\left(M_{1}, h_{1}\right)$ are said to be concordant if there is a locally flat embedding

$$
e:\left(M_{0} \times I, M_{0} \times 0, M_{0} \times 1\right) \rightarrow\left(S^{n} \times I, M_{0}, M_{1}\right)
$$

and a homotopy

$$
H_{t}: K \rightarrow M_{0}
$$

such that

- $e$ restricted to $M_{0} \times 0$ is the inclusion and $e$ maps $M_{0} \times 1$ homeomorphically onto $M_{1}$;

- $H_{0}=h_{0}$ and $H_{1}$ followed by $e(\cdot, 1)$ coincides with $h_{1}$.

Let

$$
E\left(K, S^{n}\right)
$$

denote the set of concordance classes of embeddings up to homotopy of $K$ in $S^{n}$. Unless confusion arises, we refer to embeddings up to homotopy as embeddings.

Received by the editors October 29, 2003 and, in revised form, May 1, 2004.

2000 Mathematics Subject Classification. Primary 55P25; Secondary 57Q35.

The author was partially supported by NSF Grant DMS-0201695.

(C)2005 American Mathematical Society Reverts to public domain 28 years from publication 
Constraints. We fix throughout integers

$$
k, n, r
$$

satisfying

$$
0 \leq k \leq n-3, \quad r \geq 1, \quad \text { and } \quad n \geq 6 .
$$

If $n \leq 7$, we also assume $k-r \geq 2$.

In addition to these constraints, we consider the inequalities

$$
\begin{aligned}
r & \geq \max \left(\frac{1}{2}(2 k-n), 3 k-2 n+2\right), \\
r & \geq \max \left(\frac{1}{2}(2 k-n+1), 3 k-2 n+3\right) .
\end{aligned}
$$

The inequalities can be interpreted as follows: the integer $r$ will be the connectivity of the space to be embedded. Consider maps from manifolds of dimension $k$ to $S^{n}$. Then, roughly, the inequalities represent the demand that the connectivity $r$ exceeds both the generic dimension of the triple point set and also, one half the generic dimension of the double point set.

Main results. Let

$$
\alpha: \mathbb{Z}_{2} \rightarrow \mathrm{GL}_{1}(\mathbb{R})
$$

denote the sign representation. If $s, t \geq 0$ are integers, let $S^{t \alpha+s}$ denote the one point compactification of the direct sum of $t$ copies of $\alpha$ with $s$-copies of the trivial representation. This is a sphere of dimension $t+s$ having a based action of $\mathbb{Z}_{2}$.

If $X$ and $Y$ are based spaces, we let $F^{\text {st }}(X, Y)$ denote the spectrum of stable maps from $X$ to $Y$ (the $j$-th space of this spectrum is the function space of maps from $X$ to $Q\left(\Sigma^{j} Y\right)$, where $Q$ denotes the stable homotopy functor).

If $X$ and $Y$ are based $\mathbb{Z}_{2}$-spaces, then $F^{\text {st }}(X, Y)$ comes equipped with the structure of a naive $\mathbb{Z}_{2}$-spectrum by conjugating functions. Let $F^{\text {st }}(X, Y)_{h \mathbb{Z}_{2}}$ denote the associated homotopy orbit spectrum.

Choose a basepoint for $K$. We consider the case when $X=K \wedge K$ with permutation action and $Y=S^{(n-1) \alpha+1} \wedge K$ with the diagonal action (where $\mathbb{Z}_{2}$ acts trivially on $K$ ).

Theorem A (Existence). Assume $K$ is $r$-connected and $\operatorname{dim} K \leq k$. There is an obstruction

$$
\theta_{K} \in \pi_{0}\left(F^{\mathrm{st}}\left(K \wedge K, S^{(n-1) \alpha+1} \wedge K\right)_{h \mathbb{Z}_{2}}\right),
$$

depending only on the homotopy type of $K$, whose vanishing is a necessary condition for $E\left(K, S^{n}\right)$ to be non-empty. If the inequality (1I) holds, then the vanishing of $\theta_{K}$ implies that $E\left(K, S^{n}\right)$ is non-empty.

Remarks. When $K$ is $(2 k-n)$-connected, the obstruction group is trivial, so there is an embedding of $K$ in $S^{n}$. Thus we recover the Stallings-Wall embedding theorem (see [Wa1, St]).

When $K$ is $(2 k-n-1)$-connected, the obstruction group is isomorphic to

$$
H^{2 k}\left(K \times K ; \pi_{2 k-n}(K)\right) /(1-T),
$$

where $T$ is the involution on $H^{2 k}\left(K \times K ; \pi_{2 k-n}(K)\right)$ given by $t \circ E$, where $E$ switches the factors of $K \times K$, and $t$ is the involution of $\pi_{2 k-n}(K)$ given by multiplication by $(-1)^{n-1}$.

This abelian group appears in the work of Habegger [Ha], who gave necessary and sufficient conditions for finding embeddings in the fringe dimension beyond 
the Stallings-Wall range. Habegger defined his obstruction using PL intersection theory. By contrast, our result will be derived homotopy theoretically using a theorem of Connolly and Williams $\mathrm{C}-\mathrm{W}$.

A recent paper of Lambrechts, Stanley and Vandembroucq [L-S-V] gives sufficient criteria for embedding 2-cones (the mapping cone of a map between suspensions) in the sphere. A related paper of Cornea [Cor] defines necessary obstructions to embedding finite complexes in the sphere. The connection between these papers and the current work is a mystery to be resolved.

Theorem B (Enumeration). Let $K$ be as above. Fix a basepoint in $E\left(K, S^{n}\right)$. Then there is a function

$$
\phi_{K}: E\left(K, S^{n}\right) \rightarrow \pi_{0}\left(F^{\mathrm{st}}\left(K \wedge K, S^{(n-1) \alpha} \wedge K\right)_{h \mathbb{Z}_{2}}\right)
$$

which is onto if inequality (11) holds. If inequality (2) holds, then $\phi_{K}$ is also oneto-one.

Corollary C (Group Structure). Assume $E\left(K, S^{n}\right)$ is non-empty. If inequality (21) holds, then $E\left(K, S^{n}\right)$ has the structure of an abelian group.

Remark. There is currently no explicit geometric description of this group structure. It would be both interesting and useful to have one.

The above results have corollaries which are too numerous to list in this introduction (see $\S \S 5-7$ ). For example, here is a consequence of Theorem B which appears to be new (cf. Corollary 6.7 below).

Corollary D (Isotopy Finiteness). In the range of inequality (2), an r-connected closed PL manifold $M^{k}$ with trivial Betti number $b_{2 k-n+1}(M)$ admits only finitely many locally flat embeddings in $S^{n}$ up to isotopy.

Outline. In $\S 2$ we recall the statement of the Connolly-Williams classification theorem. In $\S 3$ we prove Theorem B In $\S 4$ we prove Theorem $\mathrm{A}$ by modifying the proof of Theorem $\mathrm{B} \S 5$ contains applications to embeddings of two cell complexes (these are already in the literature in some form). In $\S 6$ we give applications to embeddings of Poincaré spaces and manifolds (many of the results in this section are new). In $\S 7$ we show that the obstructions to embedding in the range of inequality (11) are 2-local.

Conventions. We work within the category of compactly generated spaces. Products are to be re-topologized using the compactly generated topology. A space is homotopy finite if it is the retract of a finite cell complex.

A non-empty space $X$ is $r$-connected if its homotopy vanishes in degrees $\leq r$ for every choice of basepoint. By convention, the empty space is (-2)-connected. A map $X \rightarrow Y$ is $r$-connected if its homotopy fiber at every basepoint is $(r-1)$ connected. A weak equivalence is a map which is $r$-connected for every $r$.

We write $\operatorname{dim} X \leq n$ if $X$ is weak equivalent to a cell complex having cells of dimension at most $n$.

\section{The Connolly-Williams Classification theorem}

We unearth a result of Connolly and Williams which relates $E\left(K, S^{n}\right)$ to a desuspension question. 
For a 1-connected homotopy finite space $K$, consider the set of pairs $(C, \alpha)$ where $C$ is a 1 -connected homotopy finite space and

$$
\alpha: S^{n} \rightarrow K * C
$$

is a map $(*=$ join $)$ which induces, via the slant product, an isomorphism in reduced singular (co-)homology $\tilde{H}^{*}(K) \cong \tilde{H}_{n-*-1}(C)$. Introduce an equivalence relation on such pairs by declaring $(C, \alpha) \sim\left(C^{\prime}, \alpha^{\prime}\right)$ if (and only if) there is a homotopy equivalence of spaces $g: C \rightarrow C^{\prime}$ satisfying $\left(\operatorname{id}_{K} * g\right) \circ \alpha \simeq \alpha^{\prime}$. Call the resulting set of equivalence classes $S W_{n}(K)$.

There is an evident map of sets

$$
E\left(K, S^{n}\right) \rightarrow S W_{n}(K)
$$

which assigns to an embedding $(M, h)$ of $K$ the complement of a choice of regular neighborhood of $M$ together with its Spanier-Whitehead duality pairing.

Theorem 2.1 (Connolly-Williams $[\mathrm{C}-\mathrm{W}])$. Assume that $K$ is $r$-connected $(r \geq 1)$ and $\operatorname{dim} K \leq k$. Furthermore, assume $k \leq n-3, n \geq 6$ and $2(k-r) \leq n$; if $n \leq 7$ assume $k-r \leq 2$. Then

$$
E\left(K, S^{n}\right) \rightarrow S W_{n}(K)
$$

is onto. If, in addition, $2(k-r) \leq n-1$, then the map is one-to-one.

Remarks. This result is not really a "classification" of embeddings, since $S W_{n}(K)$ has not been determined. We will be concerned with the problem of computing $S W_{n}(K)$ when additional constraints are present.

The result requires $n \geq 6$ because surgery theory is used in the proof. A Poincaré embedding version of the result also holds without the requirement $\geq 6$ or additional conditions in dimensions $\leq 7$. The Poincaré version can be proved with the fiberwise homotopy-theoretic techniques appearing in [K12]. I intend to give a proof of the Poincaré version in a future paper.

A variant. We describe a variant of $S W_{n}(K)$ which is more convenient to work with. Assume that $K$ is equipped with a basepoint.

Let $\mathcal{D}_{n-1}(K)$ be defined as follows: consider the set of pairs $(W, \alpha)$ such that $W$ is a based space and $\alpha: S^{n-1} \rightarrow K \wedge W$ is a stable $S$-duality map. Define an equivalence relation by $(W, \alpha) \sim\left(W^{\prime}, \alpha^{\prime}\right)$ if and only if there is an (unstable, based) map $g: W \rightarrow W^{\prime}$ such that $\left(\operatorname{id}_{K} \wedge g\right) \circ \alpha \simeq \alpha^{\prime}$.

Lemma 2.2. Assume that $K$ is $r$-connected $(r \geq 1)$, $\operatorname{dim} K \leq k$ and $k \leq n-3$. Then there is a function

$$
\phi: S W_{n}(K) \rightarrow \mathcal{D}_{n-1}(K)
$$

which is onto if $2(k-r) \leq n+1$. If $2(k-r) \leq n$, then $\phi$ is also one-to-one.

Proof. Let $(C, \alpha)$ be a representative of $S W_{n}(K)$. Choose a basepoint for $C$. There is a well-known natural weak equivalence

$$
K * C \stackrel{\sim}{\rightarrow} \Sigma K \wedge C .
$$

Precomposing this weak equivalence with the map $\alpha$, we obtain a map $S^{n} \rightarrow \Sigma K \wedge C$ which we can arrange to be a based map by precomposing with a suitable rotation. The associated stable map $S^{n-1} \rightarrow K \wedge C$ is an $S$-duality. We leave it to the reader to check that $\phi$ is well defined. 
We now check that $\phi$ is onto. Let $(W, \alpha)$ respresent an element of $\mathcal{D}_{n-1}(K)$. Then $\alpha: S^{n-1} \rightarrow K \wedge W$ is a stable $S$-duality map. It follows that $\tilde{H}_{*}(W) \cong$ $\tilde{H}^{n-*-1}(K)=0$ if $n-*-1>k$. Thus $W$ has vanishing homology when $* \leq n-k-2$. In particular, as $k \leq n-3$, it follows that $H_{1}(W)=0$.

Let $i: W \rightarrow W^{+}$be the natural map to the (Quillen) plus construction. Then $W^{+}$is 1 -connected and we have

$$
(W, \alpha) \sim\left(W^{+},\left(\operatorname{id}_{K} \wedge i\right) \circ \alpha\right) .
$$

Using $S$-duality, it is also straightforward to check that $W^{+}$is homotopy finite. Consequently, we are entitled to assume without loss in generality that $W$ is 1 connected and homotopy finite.

In fact, the above argument shows that $W$ is $(n-k-2)$-connected. We infer that the smash product $\Sigma K \wedge W$ is $(n-k+r)$-connected. By the Freudenthal suspension theorem, the stable map $S^{n-1} \rightarrow K \wedge W$ is represented by an unstable map $\beta: S^{n} \rightarrow$ $\Sigma K \wedge W$ when $2(k-r) \leq n+1$ (unique up to homotopy if $2(k-r) \leq n$ ). This shows that the function $\phi$ is onto if $2(k-r) \leq n+1$. This argument also shows that $\phi$ is one-to-one if $2(k-r) \leq n$.

Corollary 2.3. The statement of Theorem 2.1 holds when $S W_{n}(K)$ is replaced by $\mathcal{D}_{n-1}(K)$.

\section{Proof of Theorem B}

Theorem $\mathrm{B}$ will follow from an enumeration result for suspension spectra appearing in [K11]. We first review the statement of this result.

Fix a 1-connected spectrum $E$. For technical reasons, we shall assume that $E$ is an $\Omega$-spectrum and that the spaces of the spectrum $E_{j}$ are cofibrant (i.e., retracts of cell complexes). Consider the set of pairs

$$
(X, h)
$$

such that $X$ is a based space and $h: \Sigma^{\infty} X \rightarrow E$ is a weak (homotopy) equivalence. Define

$$
(X, h) \sim(Y, g)
$$

if there is a map $f: X \rightarrow Y$ such that $g \circ \Sigma^{\infty} f$ is homotopic to $h$ (in particular, $f$ is a homology isomorphism). This generates an equivalence relation. Let $\Theta_{E}$ denote the associated set of equivalence classes.

We write $\operatorname{dim} E \leq k$ if $E$ can be obtained from the trivial spectrum by attaching cells of dimension $\leq k$. Recall that the second extended power $D_{2}(E)$ is the homotopy orbit spectrum of $\mathbb{Z}_{2}$ acting on $E^{\wedge 2}$.

Theorem 3.1 (Klein Kl1]). Assume $\Theta_{E}$ is nonempty and is equipped with a choice of basepoint. Then there is a basepoint preserving function

$$
\phi: \Theta_{E} \rightarrow\left[E, D_{2}(E)\right] .
$$

If $E$ is $r$-connected, $r \geq 1$ and $\operatorname{dim} E \leq 3 r+2$, then $\phi$ is a surjection. If in addition $\operatorname{dim} E \leq 3 r+1, \phi$ is a bijection. 
3.1. Recall that

$$
F^{\mathrm{st}}\left(K, S^{n-1}\right)
$$

is the spectrum of stable maps from $K$ to $S^{n-1}$.

Lemma 3.2. There is a bijection

$$
\Theta_{F^{\text {st }}\left(K, S^{n-1}\right)} \cong \mathcal{D}_{n-1}(K) .
$$

Proof. An element of $\Theta_{F^{\text {st }}\left(K, S^{n-1}\right)}$ is represented by a pair $(C, \alpha)$, where $C$ is a based space and $\alpha: \Sigma^{\infty} C \rightarrow F^{\text {st }}\left(K, S^{n-1}\right)$ is a weak equivalence. Taking the adjunction, this is the same as specifying a (stable) $S$-duality map $\alpha: C \wedge K \rightarrow S^{n-1}$. A standard application of $S$-duality (the "umkehr" or transpose map) then allows us to associate to $\alpha$ an $S$-duality map $\alpha^{*}: S^{n-1} \rightarrow K \wedge C$. The pair $\left(C, \alpha^{*}\right)$ then represents an element of $\mathcal{D}_{n-1}(K)$. It is straightforward to check that this procedure defines a bijection.

Lemma 3.3. Let $E=F^{\mathrm{st}}\left(K, S^{n-1}\right)$. Then there is an isomorphism of abelian groups

$$
\left[E, D_{2}(E)\right] \cong \pi_{0}\left(F^{\mathrm{st}}\left(K \wedge K, S^{(n-1) \alpha} \wedge K\right)_{h \mathbb{Z}_{2}}\right) .
$$

Proof. Note that $E \simeq K^{*} \wedge S^{n-1}$, where $K^{*}=F^{\text {st }}\left(K, S^{0}\right)$ is the $S$-dual of $K$. For spectra $A$ and $B$, let $F(A, B)$ denote the associated function spectrum. Then $\pi_{0}(F(A, B))=[A, B]$.

The first step is to rewrite

$$
F\left(E, D_{2}(E)\right) \simeq F(E, E \wedge E)_{h \mathbb{Z}_{2}}
$$

(the $\mathbb{Z}_{2}$-action on $F(E, E \wedge E)$ is induced by a permutation action on the smash product $E \wedge E$ ). There will be such an equivalence for any homotopy finite spectrum $E$. To see this, note there is a natural map from right to left: explicitly, there is a map $q_{\#}: F(E, E \wedge E) \rightarrow F\left(E, D_{2}(E)\right)$ induced by the evident map $q: E \wedge E \rightarrow$ $D_{2}(E)$. When we give $F\left(E, D_{2}(E)\right)$ the trivial $\mathbb{Z}_{2}$-action, the map $q_{\#}$ becomes equivariant. So $q_{\#}$ factorizes through the map $F(E, E \wedge E) \rightarrow F(E, E \wedge E)_{h \mathbb{Z}_{2}}$. Hence, we have a map $F(E, E \wedge E)_{h \mathbb{Z}_{2}} \rightarrow F\left(E, D_{2}(E)\right)$. It is evident that this map is an equivalence when $E$ is a sphere. One can show that the map is a weak equivalence for all homotopy finite $E$ by induction on a cell structure (we omit the details). Another more direct way to see the equivalence is to use $S$-duality to identify the domain with $E^{*} \wedge D_{2}(E)$ and the codomain with $\left(E^{*} \wedge(E \wedge E)\right)_{h \mathbb{Z}_{2}}$, where $E^{*}:=F\left(E, S^{0}\right)$ is the $S$-dual of $E$. As $\mathbb{Z}_{2}$ acts trivially on $E^{*}$, we obtain the desired equivalence.

Substituting the value of $E=F^{\mathrm{st}}\left(K, S^{n-1}\right)$ into the above, we get

$$
F\left(E, D_{2}(E)\right) \simeq F\left(K^{*} \wedge S^{n-1},\left(K^{*} \wedge S^{n-1}\right)^{\wedge 2}\right)_{h \mathbb{Z}_{2}} .
$$

Now, using the fact that $S^{n-1} \wedge S^{n-1}$ with permutation action is homeomorphic to $S^{(n-1) \alpha} \wedge S^{n-1}$ with diagonal action, the right side of the last display can be rewritten as

$$
F\left(K^{*}, S^{(n-1) \alpha} \wedge K^{*} \wedge K^{*}\right)_{h \mathbb{Z}_{2}} .
$$

For homotopy finite spectra $A$ and $B$, it is well known that the transpose map $F(A, B) \rightarrow F\left(B^{*}, A^{*}\right)$ is a weak equivalence. Consequently, there is a $\mathbb{Z}_{2}$-equivariant weak equivalence of spectra

$$
F^{\text {st }}\left(K \wedge K, S^{(n-1) \alpha} \wedge K\right) \simeq F\left(K^{*}, S^{(n-1) \alpha} \wedge K^{*} \wedge K^{*}\right),
$$

given by the transpose map. 
Taking homotopy orbits of this last equivalence, and assembling the prior information, we conclude that there is a weak equivalence of spectra

$$
F\left(E, D_{2}(E)\right) \simeq F^{\mathrm{st}}\left(K \wedge K, S^{(n-1) \alpha} \wedge K\right)_{h \mathbb{Z}_{2}} .
$$

Applying $\pi_{0}$ to this last equivalence completes the proof.

To complete the proof of Theorem B, one just needs to apply Corollary 2.3 Lemma 3.2. Lemma 3.3 and Theorem 3.1 in the stated order (to apply 3.1, use the fact that $E=F^{\mathrm{st}}\left(K, S^{n-1}\right)$ is $(n-k-2)$-connected and $\left.\operatorname{dim} E \leq n-r-2\right)$. We leave it to the reader to check that the inequalities listed in the statement of Theorem B suffice to apply these results.

\section{Proof of Theorem $\mathrm{A}$}

The proof of Theorem $\mathrm{A}$ is almost identical to the proof of Theorem $\mathrm{B}$, There are two essential differences: the first is that instead of using Theorem 3.1 we need to use the following existence result for realizing a spectrum as a suspension spectrum in the metastable range:

Theorem 4.1 (Klein [K11]). There is an obstruction

$$
\delta_{E} \in\left[E, \Sigma D_{2}(E)\right]
$$

(depending only on the homotopy type of $E$ ) which is trivial whenever $E$ has the homotopy type of a suspension spectrum.

Conversely, if $E$ is r-connected, $r \geq 1$ and $\operatorname{dim} E \leq 3 r+2$, then $E$ has the homotopy type of a suspension spectrum if $\delta_{E}=0$.

The second essential difference is that when $E=F^{\text {st }}\left(K, S^{n-1}\right)$, we have an isomorphism of abelian groups

$$
\left[E, \Sigma D_{2}(E)\right] \cong F^{\mathrm{st}}\left(K \wedge K, S^{(n-1) \alpha+1} \wedge K\right)_{h \mathbb{Z}_{2}} .
$$

The obstruction $\theta_{K}$ is defined so as to correspond to the obstruction $\delta_{E}$ with respect to this isomorphism of abelian groups. We omit the details.

\section{Applications to two Cell complexes}

Existence. It seems that the case of embedding complexes with two cells was first considered by Cooke [Co1 (see also [Co2]) and later by Connolly and Williams C-W, §5].

Let $K=S^{p} \cup_{f} e^{q+1}$ be a two cell complex, where $f: S^{q} \rightarrow S^{p}$ is some map. Let $E:=F^{\text {st }}\left(K, S^{n-1}\right)$ denote the stable Spanier-Whitehead $(n-1)$-dual of $K$. Set $p^{\prime}=n-p-2$ and $q^{\prime}=n-q-2$.

Then $E$ is the homotopy cofiber of a stable umkehr map

$$
f^{*}: S^{p^{\prime}} \rightarrow S^{q^{\prime}} .
$$

As stable classes in $\pi_{q-p}^{\text {st }}\left(S^{0}\right)$, we have

$$
\left[f^{*}\right]=[ \pm f] .
$$

Tracing through the definition of the umkehr map, with slightly extra care, the sign can be determined as $(-1)^{q p^{\prime}}$. 
In any case, $E$ has the homotopy type of a suspension spectrum if and only if $f^{*}$ is represented by an unstable map. In our range, this is equivalent to demanding that the James-Hopf invariant

$$
H_{2}\left(f^{*}\right)=\pi_{p^{\prime}}^{\mathrm{st}}\left(D_{2}\left(S^{q^{\prime}}\right)\right)
$$

is trivial.

Enumeration. Suppose $K=S^{p} \cup_{f} e^{q+1}$ admits an embedding in $S^{n}$. An analysis similar to the previous case shows that there is an isomorphism of based sets

$$
E\left(K, S^{n}\right) \cong \pi_{p^{\prime}+1}^{\mathrm{st}}\left(D_{2}\left(S^{q^{\prime}}\right)\right) .
$$

At the prime 2, the stable homotopy groups appearing on the right have been calculated by Mahowald in degrees $p^{\prime} \leq \min \left(3 q^{\prime}-3,2 q^{\prime}+29\right)$ (see Mahowald Ma. table 4.1]).

For example, suppose that $q^{\prime} \equiv 1 \bmod 16$. Then the first few groups are

\begin{tabular}{|c|c|c|c|c|c|c|c|}
\hline$j$ & 0 & 1 & 2 & 3 & 4 & 5 & 6 \\
\hline$\pi_{2 q^{\prime}+j}\left(D_{2}\left(S^{q^{\prime}}\right)\right)$ & $\mathbb{Z}_{2}$ & $\mathbb{Z}_{2}$ & $\mathbb{Z}_{8}$ & $\mathbb{Z}_{2}$ & 0 & $\mathbb{Z}_{2}$ & $\mathbb{Z}_{16} \oplus \mathbb{Z}_{2}$ \\
\hline
\end{tabular}

\section{Embeddings of Poincaré spaces}

In this section we assume that $K$ is an $r$-connected Poincaré duality space of formal dimension $k$.

Remarks. The Browder-Casson-Sullivan-Wall theorem ([Wa2, Th. 12.1]) says that concordance classes of Poincaré embeddings of $K$ in $S^{n}$ are in one-to-one correspondence with embeddings up to homotopy of $K$ in $S^{n}$.

If $K$ is a closed PL manifold, then [Wa2, Th. 11.3.1] implies that $E\left(K, S^{n}\right)$ is in bijection with the isotopy classes of locally flat PL embeddings of $K$ in $S^{n}$.

By [Wa2, Lem. 2.8], we can find a homotopy equivalence $K \simeq L \cup e^{k}$, where $L$ is a finite complex and $\operatorname{dim} L \leq k-r-1$. In particular, we have a cofibration sequence of $\mathbb{Z}_{2 \text {-spaces }}$

$$
L \wedge K \cup_{L \wedge L} K \wedge L \rightarrow K \wedge K \rightarrow S^{k} \wedge S^{k}
$$

The first term of this sequence has dimension $\leq 2 k-r-1$, so we may infer that the evident map

$$
F^{\mathrm{st}}\left(S^{k} \wedge S^{k}, S^{(n-1) \alpha+1} \wedge K\right)_{h \mathbb{Z}_{2}} \rightarrow F^{\mathrm{st}}\left(K \wedge K, S^{(n-1) \alpha+1} \wedge K\right)_{h \mathbb{Z}_{2}}
$$

is $(n-2(k-r)+1)$-connected. In particular, if $n \geq 2(k-r)$, we see that this map induces an isomorphism on path components.

By elementary manipulations, which we omit, there is an identification

$$
F^{\mathrm{st}}\left(S^{k} \wedge S^{k}, S^{(n-1) \alpha+1} \wedge K\right)_{h \mathbb{Z}_{2}} \simeq F^{\mathrm{st}}\left(S^{n-2}, K \wedge D_{2}\left(S^{n-k-1}\right)\right) .
$$

We conclude:

Theorem 6.1. Assume in addition $n \geq 2(k-r)$. Then the obstruction $\theta_{K}$ is detected in the abelian group

$$
\pi_{n-2}^{\mathrm{st}}\left(K \wedge D_{2}\left(S^{n-k-1}\right)\right)
$$


Remark. Let $\nu$ be the Spivak normal fibration of $K$; we consider $\nu$ as having fiber a stable $(-k)$-sphere. Let $K^{\nu}$ denote the Thom spectrum of $\nu$. When $K$ embeds in $S^{n}$, the fibration $\nu$ compresses down to an unstable $(n-k-1)$-spherical fibration. Conversely, when $\nu$ compresses, a construction due to Browder gives an embedding of $K$ in $S^{n+1}$ (see [B]).

It is therefore tempting to try and relate $\theta_{K}$ to the obstruction-theoretic problem of finding a compression of $\nu$. We do not as yet have a solution to this.

By essentially the same argument that proves 6.1, we have

Theorem 6.2. Assume $n>2(k-r)$. Then the function $\phi_{K}$ can be rewritten as

$$
\phi_{K}: E\left(K, S^{n}\right) \rightarrow \pi_{n-1}^{\mathrm{st}}\left(K \wedge D_{2}\left(S^{n-k-1}\right)\right) .
$$

The remainder of this section is devoted to obtaining corollaries of 6.1 and 6.2 Our first result shows that $\phi_{K}$ is homological in the fringe dimension beyond the stable range.

Corollary 6.3 (Compare [H-H] Th. 2.3], [Ha]). The obstruction $\phi_{K}$ to embedding $K$ in $S^{2 k-r-1}$ lives in the abelian group

$$
H_{r+1}\left(K ; \mathbb{Z}_{s}\right),
$$

where $s=1+(-1)^{k-r+1}$.

Proof. The Hurewicz map

$$
\begin{aligned}
\pi_{2 k-r-3}^{\mathrm{st}}\left(K \wedge D_{2}\left(S^{k-r-2}\right)\right) & \rightarrow H_{2 k-r-3}\left(K \wedge D_{2}\left(S^{k-r-2}\right)\right) \\
& \cong H_{r+1}(K) \otimes H_{2(k-r-2)}\left(D_{2}\left(S^{k-r-2}\right)\right) \\
& \cong H_{r+1}\left(K ; \mathbb{Z}_{s}\right)
\end{aligned}
$$

is an isomorphism in this degree. Now apply Theorem 6.1

By a similar argument, which we omit (use 6.2), we obtain

Corollary 6.4 (Compare [H-H, Th. 2.4], [Ha $)$. The set of concordance classes of embeddings of $K$ in $S^{2 k-r+2}$ is isomorphic to

$$
H_{r+1}\left(K ; \mathbb{Z}_{s}\right),
$$

where $s=1+(-1)^{k-r}$.

Our next pair of corollaries concerns the outcome of tensoring with the rationals.

Corollary 6.5. If $n \equiv k \bmod 2$, then $\theta_{K} \otimes \mathbb{Q}$ is trivial. Otherwise, $\theta_{K} \otimes \mathbb{Q}$ is detected in the vector space $H_{2 k-n}(K ; \mathbb{Q})$.

Proof. If $n \equiv k \bmod 2$, then $\pi_{*}^{\text {st }}\left(D_{2}\left(S^{n-k-1}\right)\right) \otimes \mathbb{Q}$ is trivial. Using the skeletal filtration of $K$ and the five lemma, we infer that $\pi_{*}^{\text {st }}\left(K \wedge D_{2}\left(S^{n-k-1}\right)\right) \otimes \mathbb{Q}$ is also trivial. The first part now follows using Theorem6.1.

For the second part, note that the transfer

$$
D_{2}\left(S^{n-k-1}\right) \rightarrow\left(S^{n-k-1}\right)^{\wedge 2}
$$

is, rationally, the inclusion of a wedge summand. Smashing with $K$ and applying rational homotopy, we infer that $\pi_{n-2}^{\text {st }}\left(K \wedge D_{2}\left(S^{n-k-1}\right)\right) \otimes \mathbb{Q}$ is a summand of $\pi_{n-2}^{\text {st }}\left(K \wedge\left(S^{n-k-1}\right)^{\wedge 2}\right) \otimes \mathbb{Q}$. Over the rationals, stable homotopy coincides with homology. It follows that $\theta_{K} \otimes \mathbb{Q}$ is detected in $H_{2 k-n}(K ; \mathbb{Q})$. 
Corollary 6.6. Assume $K$ embeds in $S^{n}$. Assume inequality (2) holds. Then $E\left(K, S^{n}\right)$ is finitely generated.

If $n \equiv k \bmod 2$, then $E\left(K, S^{n}\right)$ is finite. Otherwise, $E\left(K, S^{n}\right) \otimes \mathbb{Q}$ is a direct summand of $H_{2 k-n+1}(K ; \mathbb{Q})$.

Proof of Corollary 6.6. The first part follows from Theorem 6.2 because $\pi_{n-1}^{\text {st }}\left(K \wedge D_{2}\left(S^{n-k-1}\right)\right)$ is finitely generated. The second part is proved in a manner similar to Corollary 6.5. We omit the details.

A direct consequence of Corollary 6.6 is:

Corollary 6.7. Assume the inequality (2) holds. If the Betti number $b_{2 k-n+1}(K)$ is trivial, then there are finitely many concordance classes of embeddings of $K$ in $S^{n}$.

This last result gives Corollary $\mathrm{D}$ of the introduction using the remarks about manifolds given at the beginning of this section.

\section{LOCALIZATION AT 2}

Let $K$ and $K^{\prime}$ be $r$-connected finite complexes with $\operatorname{dim} K, \operatorname{dim} K^{\prime} \leq k$.

Theorem 7.1. Suppose that $f: K \rightarrow K^{\prime}$ is a 2-local homotopy equivalence. Assume that inequality (1) holds. Then $K$ embeds in $S^{n}$ if and only if $K^{\prime}$ does.

Remark. Rigdon [Ri] and Williams Wi prove a similar result for manifolds in the metastable range $n \geq \frac{3}{2}(k+1)$. The main difference between their result and ours is that ours holds outside of the metastable range at the expense of an additional connectivity hypothesis.

Proof of Theorem 7.1. The induced map of stable (n-1)-duals

$$
E^{\prime}:=F^{\mathrm{st}}\left(K^{\prime}, S^{n-1}\right) \stackrel{f^{*}}{\rightarrow} F^{\mathrm{st}}\left(K, S^{n-1}\right)=: E
$$

is clearly a 2-local equivalence. By $\left[\mathrm{Kl1}\right.$, Th. D], $E^{\prime}$ is a suspension spectrum if and only if $E$ is. The result now follows by applying Lemmas 3.2, 2.2 and Theorem 2.1.

\section{ACKNOWLEDGEMENTS}

I wish to thank Bill Richter for introducing me to the notion of Poincaré embedding. Bill also gave me a copy of Habegger's thesis to read when I was an undergraduate in the early 1980s. I am very much indebted to Bruce Williams for introducing me to his papers on embeddings. I am also grateful to Bill and Bruce for mathematical discussions dating back more than twenty years.

\section{REFERENCES}

[B] Browder, W.: Embedding smooth manifolds. Proc. Internat. Congr. Math., (Moscow, 1966). Izdat. "Mir", Moscow 712-719 (1968) MR0238335 (38:6611)

[C-W] Connolly, F. X., Williams, B.: Embeddings up to homotopy type and geometric suspensions of manifolds. Quart. J. Math. Oxford 29, 385-401 (1978) MR0517733 (83a:57022)

[Co1] Cooke, G.: Embedding certain complexes up to homotopy type in euclidean space. Ann. of Math. 90, 144-156 (1969) MR0242152 (39:3486)

[Co2] Cooke, G.: Thickenings of CW complexes of the form $S^{m} \cup_{\alpha} e^{n}$. Trans. Amer. Math. Soc. 247, 177-210 (1979) MR.0517691 (81c:55016) 
[Cor] Cornea, O.: New obstructions to the thickening of CW complexes. Proc. Amer. Math. Soc. 132, 2769-2781 (2004) MR2054804

[H-H] Haefliger, A., Hirsch, M. W.: On the existence and classification of differentiable embeddings. Topology 2, 129-135 (1963) MR0149494(26:6981)

[Ha] Habegger, N.: Embedding up to homotopy type - the first obstruction. Topology Appl. 17, 131-143 (1984) MR0738942(85e:57022)

[Kl1] Klein, J. R.: Moduli of suspension spectra. arXiv:math.AT/0210258, to appear in Trans. Amer. Math. Soc.

[K12] Klein, J. R.: Poincaré embeddings and fiberwise homotopy theory. Topology 38, 597-620 (1999) MR1670412 (2000b:57037)

[L-S-V] Lambrechts, P., Stanley, D., Vandembroucq, L.: Embeddings up to homotopy of twocones in Euclidean space. Trans. Amer. Math. Soc. 354, 3973-4013 (2002) MR1926862 (2003h:55012)

[Ma] Mahowald, M.: The metastable homotopy of $S^{n}$. (Memoirs of the Amer. Math. Soc., Vol. 27). Amer. Math. Soc. 1967 MR0236923 (38:5216)

[Ri] Rigdon, R.: p-equivalences and embeddings of manifolds. J. London Math. Soc. 2 (1975) MR0431211 (55:4213)

[St] Stallings, J. R.: Embedding homotopy types into manifolds. 1965 unpublished paper (see http://math.berkeley.edu/ stall for a TeXed version)

[Wa1] Wall, C. T. C.: Classification problems in differential topology_IV. Thickenings. Topology 5, 73-94 (1966) MR0192509(33:734)

[Wa2] Wall, C. T. C.: Surgery on Compact Manifolds. (Mathematical Surveys and Monographs, Vol. 69). Amer. Math. Soc. 1999 MR1687388 (2000a:57089)

[Wi] Williams, B.: Hopf invariants, localizations, and embeddings of Poincaré complexes. Pacific J. Math. 84, 217-224 (1979) MF0559639(81e:57023)

Department of Mathematics, Wayne State University, Detroit, Michigan 48202

E-mail address: klein@math. wayne.edu 